\title{
A rare retrovesical hydatid cyst and value of transrectal ultrasonography in diagnosis: a case report and review of the literature
}

\author{
Emre Unal $^{1}$, Meryem Keles², Sibel Yazgan', Musturay Karcaaltincaba ${ }^{3}$
}

${ }^{1}$ Zonguldak Atatürk State Hospital, Department of Radiology, Zonguldak, ${ }^{2}$ Zonguldak Atatürk State Hospital, Deparment of Internal Medicine, Zonguldak, ${ }^{3}$ Hacettepe University, School of Medicine, Department of Radiology, Ankara, Turkey

\begin{abstract}
We present a large hydatid cyst located midline and posterior to prostatic gland and seminal vesicles in an asymptomatic patient. Computed tomography (CT) scan revealed a large retrovesical mass and the transrectal ultrasonography idetified a degenerated hydatid cyst with "ball of wool" appearance. Although extremely rare, hydatid disease should be kept in mind in the differential diagnosis of pelvic particularly retrovesical midline masses.
\end{abstract}

Keywords: hydatid cyst; rectovesical pouch; retrovesical; ball of wool sign; transrectal ultrasonography.

\section{Introduction}

Echinococcosis, also known as hydatid disease, is a common zoonotic infection caused by Echinococcus tapeworms, Echinococcus granulosus, and Echinococcus multilocularis are the main forms of hydatid disease with the former being more common [1,2]. Hydatid disease primarily affects the liver but the lung, kidney, spleen, bone, or brain can also be affected [2]. Hydatid cysts have characteristic imaging findings based on the stage of cyst, therefore it is important to discriminate inactive cysts from active disease [1,2]. Ultrasonography (US) is the main modality for initial diagnosis of hydatid disease and may also reveal viability, as well $[1,2]$.

Hydatid cysts located in retrovesical and periprostatic regions are rare entities and reported only as case studies in the literature [3-9]. Diagnosis of a retrovesical hydatid cyst should be taken into consideration particularly

Received 24.07.06.2016 Accepted 30.07.2016

Med Ultrason

2017, Vol. 19, No 1, 111-113

Corresponding author: Emre Ünal

Zonguldak Ataturk State Hospital,

Department of Radiology,

Zonguldak, 67030, Turkey

Phone: +90 372252 1900/2846-2847

Fax: +90 3722520725

E-mail: emreunal.rad@gmail.com in patients with previous history of hydatid disease. The computed tomography (CT) study can be problematic due to contents of the cyst. Therefore TR-US (transrectal ultrasonography) should be the primary initial imaging modality.

Herein, we report a 31 year-old-man with incidentally detected large inactive hydatid cyst in the rectovesical pouch.

\section{Case report}

A 31 year-old man was referred to hepatobiliary ultrasound examination to rule out cholelithiasis. The patient had nonspecific dyspeptic complaints for two weeks which resolved completely without treatment. The gallbladder and bile ducts were normal in US examination, however there was a heterogenous exophytic liver mass which was consistent with the diagnosis of degenerated hydatid cyst (fig 1 a). The patient had no history of albendazole treatment and also had no previous abdominal imaging. Abdominal CT scan supported US findings (fig $1 \mathrm{~b}$ ), however CT scan also demonstrated an additional mass located retrovesical pouch. CT findings were inconclusive for the diagnosis of hydatid cyst (fig $2 a, b)$. Subsequently TR-US was performed and clearly demonstrated another degenerated hydatid cyst located posterior to seminal vesicles (fig 2c,d). 


\section{Discussions}

Peritoneal and pelvic hydatid disease commonly result from secondary dissemination of the disease. However, there are several case reports point out that primary involvement may also occur $[3,5,6,10,11]$. Our patient had an exophytic liver hydatid cyst, therefore secondary rather than primary involvement was considered. However retrovesical hydatid cysts are extremely rare entities regardless of the pathophysiology.

Mullerian duct, prostatic utricle, and ejaculatory duct cysts are more commonly seen cystic lesions in this region [12]. However these cysts usually do not exceed 2 $\mathrm{cm}$ in size. In the presented case retrovesical hydatid cyst had a size of about 5-6 cm. Gandhiraman et al reported a case of large primary peritoneal hydatid cyst which was misdiagnosed as an ovarian cyst torsion [5]. Moreover, Abike et al reported a pelvic hydatic cyst which was thought to be an ovarian carcinoma [3]. Although there are several differential diagnosis in men regarding to ret-

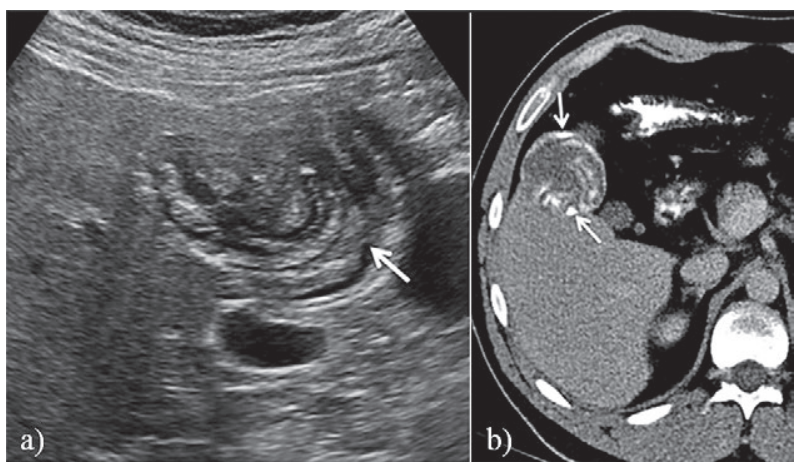

Fig 1. Inactive echinococcal cyst involving the liver: (a) axial transabdominal US and (b) post contrast CT images show degenerated exophytic liver hydatid cyst with "ball of wool" sign (arrows in a) and wall calcifications (arrows in b). rovesical cystic lesions, in women pelvic hydatid disease can be mistaken for ovarian pathologies. Detection of the ovaries separately from the mass is the key finding however such differentiation may not be possible in most cases due to large size of the mass.

It has been reported that patients with retrovesical hydatid cysts may suffer from dysuria, voiding obstruction, and abdominal pain $[4,8-10]$. However such symptoms are usually seen in the case of direct communication or due to mass effect. In the presented case retrovesical hydatid cyst was incidentally detected. Furthermore the patient was asymptomatic and there was no sign of mass effect or direct communication with urinary tract. Although surgery was recommended in aforementioned cases [4,8$10]$, in the present case hydatid cyst was inactive (type 4) and demonstrated "ball of wool" sign [1].

US should be the initial diagnostic modality for the differential diagnosis of pelvic masses. Nevertheless, US may be insufficient for deep located lesions such as retrovesical or pelvic region. However TR-US may provide excellent resolution in these circumstances as we experienced in our case. In the literature CT was reported to be more accurate than US for identification of hydatid cyst in selected cases $[3,6]$. In the presence of wall calcification or daughter cysts CT may be useful for the diagnosis of hydatid cyst. Although such findings can also be evaluated by US as well. On the other hand, magnetic resonance imaging (MRI) may clearly demonstrate hydatid cyst and presence of daughter cysts or detached membrane due to increased contrast resolution, however MRI is much more expensive than TR-US examination.

In conclusion, although it is rare the diagnosis of hydatid disease should be considered in the differential diagnosis of retrovesical masses, particularly in patients with hydatid disease located in other sites.

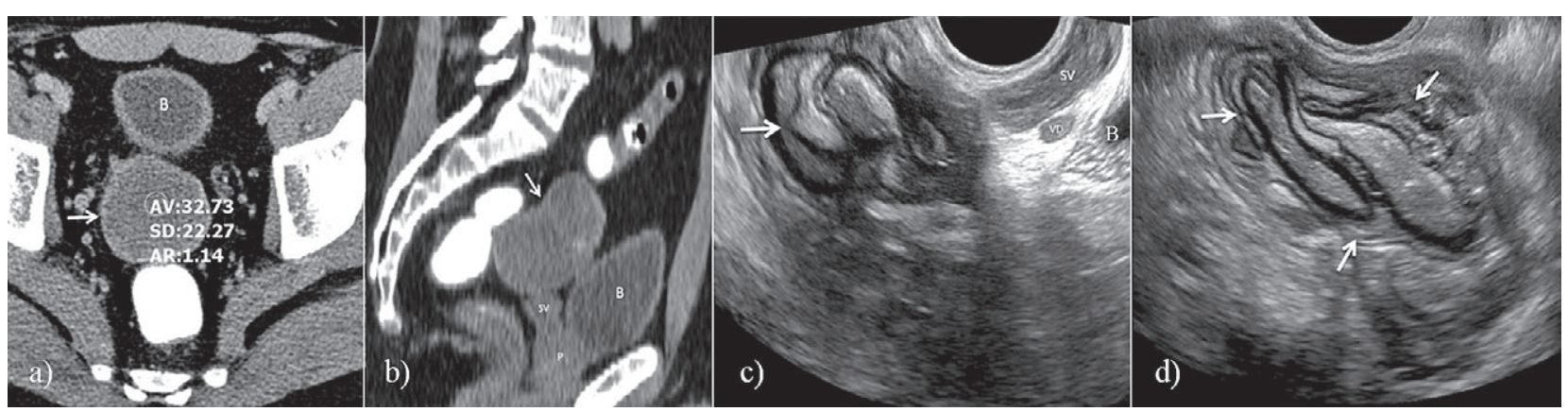

Fig 2. a) Axial post contrast CT image of the pelvis shows a non specific mass in rectovesical pouch (arrow); b) Sagittal post contrast CT image demonstrates the localisation of the mass without providing any additional information for the diagnosis; c) However, sagittal TR-US image from the same location reveals degenerated hydatid cyst (arrow) with "ball of wool" appearance. In addition, TR-US more clearly depicts the relationship of the hydatid cyst with seminal vesicles (SV), vas deferens (VD) and bladder (B); d) Axial TR-US image demonstrates "ball of wool" sign in detail (arrows). P: prostate gland. 


\section{References}

1. Czermak BV, Akhan O, Hiemetzberger R, et al. Echinococcosis of the liver. Abdom Imaging 2008;33:133-143.

2. Pedrosa I, Saiz A, Arrazola J, Ferreiros J, Pedrosa CS. Hydatid disease: radiologic and pathologic features and complications. Radiographics 2000; 20:795-817.

3. Abike F, Dunder I, Tapisiz OL, et al. Primary pelvic hydatic cyst mimicking ovarian carcinoma. J Chin Med Assoc 2011;74:237-239.

4. Boukadoum N, Bensoltane S, Chettibi K, Yassi F, Kadi A. Prostatic hydatid cyst: a case study. Diagn Interv Imaging 2012;93:793-795.

5. Gandhiraman K, Balakrishnan R, Ramamoorthy R, Rajeshwari R. Primary Peritoneal Hydatid Cyst Presenting as Ovarian Cyst Torsion: A Rare Case Report. J Clin Diagn Res 2015; 9:QD07-08.

6. Halefoglu AM, Yasar A. Huge retrovesical hydatid cyst with pelvic localization as the primary site: a case report Acta Radiol 2007;48:918-920.
7. Ozer T, Gundogdu S, Ozer Y, Mahmutyazicioglu K, Savranlar A, Ozdemir H. Echinococcosis involving the liver, retrovesical and seminal vesicle presented with syncope. Int J Urol 2004;11:922-924.

8. Papathanasiou A, Voulgaris S, Salpiggidis G, Charalabous S, Fatles G, Rombis V. Hydatid cyst of the seminal vesicle. Int J Urol 2006;13:308-310.

9. Tuygun C, Bakirtas H, Imamoglu MA, Sertcelik N, Zengin $\mathrm{K}$, Bozkurt IH. The unusual mass of retrovesical space: a secondary hydatid cyst disease. ScientificWorldJournal 2006;6:2481-2485.

10. Ercil H, Gurlen G, Sener NC, Altunkol A, Kuyucu F, Evliyaoglu Y. A rare cause of lower urinary tract symptoms: retrovesical hydatid cyst. J Pak Med Assoc 2014;64:10871089.

11. Sallami S, Nouira Y, Rhouma SB, et al. Endoscopic treatment of a primary prostatic hydatid cyst: a mini-invasive therapeutic approach. Urol J 2010;7:270-274.

12. Nghiem HT, Kellman GM, Sandberg SA, Craig BM. Cystic lesions of the prostate. Radiographics 1990;10:635-650. 